\title{
Nachrichten
}

Städte arbeiten weiter an der Modernisierung ihrer Verwaltungen

Trotz großer finanzieller Engpässe, erheblicher Arbeitsbelastung durch die Umsetzung der Reformen am Arbeitsmarkt und trotz fortgesetzten Personalabbaus verfolgen die deutschen Städte konsequent das Ziel, die Strukturen und Abläufe in ihren Verwaltungen zu modernisieren. Das zeigen die Ergebnisse einer Umfrage zum Stand der Verwaltungsmodernisierung, die der Deutsche Städtetag (DST) und das Deutsche Institut für Urbanistik (Difu) unter den Mitgliedsstädten des Deutschen Städtetages durchgeführt haben.

Die Veröffentlichung der Umfrageergebnisse kann im Buchhandel bezogen oder per Post beim Deutschen Institut für Urbanistik, Postfach 1203 21, 10593 Berlin, Fax (030) 39001-275, EMail: vertrieb@difu.de, bestellt werden. Bibliographische Angaben: »Verwaltungsmodernisierung in deutschen Kommunalverwaltungen - Eine Bestandsaufnahme«, Bearbeiter: Rüdiger Knipp. Bd. 6/2005, 180 S., 37 Abb., umfangreicher Tabellenanhang, Schutzgebühr 20 Euro, ISBN 3-88118-367-1.

\section{CAFM in der Kirche}

Der Trend zu einem gezielten Immobilienmanagement mit informationstechnischer Unterstützung aller damit verbundenen Prozesse macht auch vor der Kirche nicht halt. So hat sich aktuell das Bistum Hildesheim dafür entschieden, seine Gebäude und Liegenschaften mit dem CAFM-System IMSware zu verwalten.

Das Bistum Hildesheim - der bischöfliche Stuhl und die Kirchengemeinden - besitzt einen Immobilienbestand von rund 2.000 Gebäuden mit einer Nutzfläche von circa 820.000 Quadratmetern. Bei dem überwiegenden Teil dieser Gebäude handelt es sich um Kirchen, Pfarrhäuser, Kindergärten, Schulen und Heime. Nachdem die vorhandenen Daten aus Programmen wie Access und Excel übernommen worden sind, soll IMSware sämtliche im Zusammenhang mit den Immobilien stehenden Informationen und Prozesse im Bistum steuern. Die Software wird dabei in erster Linie für das Projekt- und Budgetmanagement - hier vor allem für die Verfolgung von Baumaßnahmen - sowie für die Bereiche Erbpacht, Mietverwaltung und das Dokumentenwesen zum Einsatz kommen.

Weitere Informationen im Internet unter: www.ismware.de.

\section{Eletronische Zulassung von Pflanzenschutzmitteln}

E-Government und Verwaltungsmodernisierung mit MACH Software lösen beim Bundesamt für Verbraucherschutz und Lebensmittelsicherheit (BVL) Papierakten ab. Das Bundesamt verabschiedet sich von der papiergebundenen Bearbeitung und startet unter dem Dach der Initiative BundOnline 2005 in das Thema EGovernment. In einem Pilotprojekt wird zunächst das Zulassungsverfahren für Pflanzenschutzmittel (gemäß $§ 15$ PflSchG) vollständig elektronisch realisiert. Die Antragstellung inklusive elektronischer Signatur und die Abwicklung des Zahlungsverkehrs für die anfallenden Gebühren werden künftig online möglich sein. Auch die Antragsbearbeitung und Aktenführung erfolgen dann vollkommen IT-gestützt - ebenso die Kommunikation mit den externen Prozessbeteiligten, also Antragstellern und Bewertungsbehörden.

Weitere Informationen im Internet unter: www.mach.de.

\section{Ostthüringen mit eGovernment-Plattform}

Für die Verwaltungen, Bürger und Unternehmen in der Region Ostthüringen werden in Zukunft viele Wege kürzer und schneller sein. Unter Federführung der Curiavant Internet GmbH, Nürnberg wird an der Umsetzung einer an der Zukunft ausgerichteten »Regionalen Internetplattform Ostthüringen « gearbeitet. Basis für diese Plattform bilden die Formular-Management-Lösung der bol Systemhaus GmbH, das Content-Management-System der Firma Six Offene Systeme GmbH und die Portalkomponenten für Authentifizierung, elektronische Signatur, ePayment und Webkatalog der Curiavant Internet $\mathrm{GmbH}$.

Ziel ist, die Internetauftritte der Städte Altenburg, Gera, Jena und des Landkreises Altenburger Land zu vernetzen und so Mehrwerte und Synergien zu schaffen. Darüber hinaus sollen alle beteiligten Verwaltungen, Bürger und Unternehmen gezielt auf die für sie relevanten Informationen zugreifen sowie attraktive Dienstleistungen und Anwendungen nutzen können. Die Region Ostthüringen realisiert so eine umfassende Internetplattform für die medienbruchfreie Kommunikation, Interaktion und Transaktion zwischen Verwaltung, Bürger und Unternehmen.

Weitere Informationen: Curiavant Internet $\mathrm{GmbH}$, Silke Abel, Hauptmarkt 17, 90403 Nürnberg, Telefon: (0911) 2318600, E-Mail: presse@curiavant.de, Internet: www.curiavant.de.

\section{Barrierefreie Webseiten}

Um allen Bürgern den Zugang zu Informationen gleichermaßen zu ermöglichen, hat der deutsche Gesetzgeber Richtlinien zum barrierefreien Internet entwickelt. Diese sind im »Gesetz zur Gleichstellung behinderter Menschen« (BGG) enthalten. Gemäß der Barrierefreien Informationstechnik-Verordnung (BITV) des BGG, einem Kriterienkatalog mit 14 verbindlichen Richtlinien, sind alle Bundesbehörden verpflichtet, bis Ende 2005 ihren Internet-Auftritt barrierefrei zu gestalten. Neue oder in wesentlichen Teilen veränderte Web-Angebote müssen ab sofort diesen Richtlinien entsprechen. Die Verordnung besagt, dass alle Inhalte und das Design der Website so gestaltet sein müssen, dass jeder Anwender sie uneingeschränkt - und prinzipiell ohne fremde Hilfe - nutzen kann.

Die E-Government Initiative BundOnline bietet allen Bundesbehörden die Content-Management-Lösung Government Site Builder an, die von MATERNA und dem Bundesverwaltungsamt entwickelt wurde. Diese Lösung ermöglicht die Erstellung barrierefreier Websites gemäß der Anforderungen des BITV. Die Funktionen rund um Layout, Bildelemente, Multimedia-Informationen, Navigation und Download-Möglichkeiten sind entsprechend dieser Anforderungen gestaltet.

Weitere Informationen: Materna GmbH, Christine Siepe, Voßkuhle 37, 44141 Dortmund, Telefon (0231) 5599-168, E-Mail: christine.siepe@materna.de, Internet: www.materna.de.

\section{Elektronische Beschaffung in der Verwaltung}

Der elektronische Einkauf spielt für öffentliche Einrichtungen eine immer wichtigere Rolle: Das zeigt die aktuell veröffentlichte EProcurement-Studie der MFG Baden-Württemberg im Rahmen der europäischen Initiative EPROC. Vor allem bei der Abwicklung von Bestellvorgängen kommt die elektronische Beschaffung zum Einsatz: 43,5 Prozent der Befragten setzen hier bereits Lösungen ein oder planen deren Einsatz.

Die Ergebnisse der Befragung sind in im aktuellen Band $» \mathrm{E}-$ Procurement in der öffentlichen Verwaltung « aus der Reihe »Stuttgarter Beiträge zur Medienwirtschaft« veröffentlicht. Die kostenlose Publikation steht unter www.doIT-online.de/sb zum Download bereit oder kann bestellt werden unter research@ $m f g . d e$. Der Sammelband stellt darüber hinaus erfolgreiche kommunale Praxisbeispiele vor und informiert in unterschiedlichen Expertenbeiträgen über die organisatorischen Voraussetzungen des elektronischen Einkaufs. 


\section{BundOnline 2005 am Ziel}

Die Initiative BundOnline 2005 wurde im Jahr 2000 mit dem Ziel gestartet, alle internetfähigen Dienstleistungen der Bundesverwaltung bis Ende des Jahres 2005 im Internet zur Verfügung zu stellen. Mit den am 29. August freigeschalteten vier Angeboten der Bundesversicherungsanstalt für Angestellte ist für Bundesinnenminister Otto Schily dieses Ziel erreicht: »Die Bürgerinnen und Bürger können heute 379 Online-Leistungen der Bundesverwaltung nutzen. Innerhalb von fünf Jahren haben wir die Dienstleistungen der Bundesverwaltung digitalisiert und im Internet verfügbar gemacht. Das E-Government-Programm hat einen gewaltigen Modernisierungsschub quer durch die gesamte Bundesverwaltung ausgelöst. Über hundert verschiedene Behörden sind mit ihren Dienstleistungen an BundOnline beteiligt.«

\section{Leasing-Handbuch}

Das »Leasing-Handbuch für die öffentliche Hand«, herausgegeben von Dr. Michael Kroll, ist als vollständig überarbeitete und aktualisierte 10. Auflage erschienen. Ausgabe 2005, 344 Seiten, 54,90 Euro, ISBN 3-931362-09-4, Bezugsadresse: LeaSoft GmbH - Fax: (09571) 6013 - Rotenhahnstraße 5, 96215 Lichtenfels.

\section{Web-basierte Personalkostenhochrechnung}

Das Software- und Beratungshaus MACH erweitert sein integriertes Produktangebot für das Verwaltungsmanagement um eine vollständig Web-basierte Lösung für die Personalkostenhochrechnung. Die Software ermöglicht sowohl eine komfortable Bedarfsrechnung für das laufende Geschäftsjahr, als auch eine Prognoserechnung für die kommenden drei bis fünf Jahre. Schnittstellen zu Personalabrechnungssystemen sind hierbei bereits realisiert, neue können mit vergleichsweise geringem Aufwand umgesetzt werden.

Integriert ist die neue Anwendung in das Web Personalmanagement, einer modernen Softwarelösung für die strategische und operative Personalarbeit im Public Sector. Die bereits bestehenden Möglichkeiten, mitarbeiter- und stellenbezogene Informationen kostenseitig auszuwerten und darzustellen, werden mit der neuen Web Personalkostenhochrechnung hinsichtlich Datenbasis und Auswertungsmöglichkeiten ausgebaut und stärker differenziert.

Weitere Informationen: MACH AG, Jochen Michels, Wielandstraße 14, 23558 Lübeck, Telefon: (0451) 70647-271, E-Mail: jmi@mach.de, Internet: www.mach.de.

\section{Tage eGovernment Good Practice Framework}

Die Europäische Kommission, GD Informationsgesellschaft und Medien, führt eine Reihe von Maßnahmen durch, um eGovernment auf allen Verwaltungsebenen der EU zu unterstützen. Das eGovernment Good Practice Framework (GPF) spielt im Zusammenhang der Initiativen der Kommission (eEurope 2005 und i2010 Information Society) eine strategisch herausragende Rolle. Das GPF dokumentiert beispielhafte eGovernment-Fälle und macht sie durch eine Suchmaschine europaweit zugänglich. Eine moderne Verwaltung, die mit innovativen Verfahren eGovernment-Dienste anbietet, ist eine Voraussetzung zur Erreichung der Ziele der Lissabon-Strategie.

Das Internetportal des GPF ist unter www.egov-goodpractice.org verfügbar. Aufgrund seiner Attraktivität wurde es in den ersten hundert Tagen bereits intensiv von Experten und Interessierten aus über sechzig Ländern genutzt.

Die Good Practice-Datenbank ist das Kernstück des Portals. Um einen direkten Wissensaustausch zu ermöglichen, können re- gistrierte Mitglieder neben den eGovernment-Fällen ihre persönlichen Kontaktdaten veröffentlichen. Die Datenbank wächst täglich, so dass auch zu speziellen Themen immer öfter ein internationaler Überblick zu Fällen und Ansprechpartnern möglich ist. Die Angebote werden regelmäßig überprüft und aktualisiert. Ein Self-Assessment wird die Bewertung der Fälle ermöglichen und so den Standard des GPF sichern.

Die Bandbreite der Beispiele innerhalb der Datenbank reicht von eGovernment-Strategien über eGovernment-Prozesse bis hin zu einzelfallbezogenen Lösungen und Anwendungen. Dr. Rainer Graf zufolge, dem GPF-Projektmanager von Bearing Point, gibt es »bereits heute eine erstaunliche Vielfalt von hervorragenden Beispielen in der Datenbank«. Die Spannweite reicht von »einem eDemokratie-Projekt in Portugal bis hin zu einem elektronischen Steuersystem in Österreich «. Innerhalb des Portals stehen dem Nutzer verschiedene Informationsquellen zur Verfügung. Das GPF enthält Nachrichten, Publikationen, Angaben zu Veranstaltungen und Links zu anderen eGovernment-Webseiten.

Das Projekt wird von Bearing Point in Zusammenarbeit mit dem Fraunhofer IAO und Fraunhofer FOKUS durchgeführt.

\section{Seminar zum Datenschutzbeauftragten}

In dem Seminar der IT-Akademie Mainz e.V. wird den Teilnehmern vermittelt, welche Maßnahmen notwendig sind, um effektiven Datenschutz im Sinne des Gesetzes zu gewährleisten. Das Seminar behandelt Inhalte, die den Datenschutzbeauftragten helfen, den technisch-organisatorischen Datenschutz im Unternehmen zu realisieren. Ausgewählte, häufig angewendete Sicherheitsmechanismen werden erläutert. Die Schulung demonstriert bestehende Schwachstellen und Effizienzprobleme und zeigt Lösungsmöglichkeiten auf. Die Teilnehmer erhalten außerdem Verfahrenshilfen für zukünftige Aufgaben an die Hand.

Information und Anmeldung: IT-Akademie Mainz e.V., Wilh.Theodor-Römheld-Str. 34, 55130 Mainz, Herr Grünberg, Telefon: (06131) 97236-31, E-Mail: gruenberg@ita-mainz.de, www.itamainz.de.

\section{Vorschau auf die kommenden Hefte}

Helmut Klages: Entbürokratisierung

Joachim Lohmann: Schuldenbegrenzung: Maastricht statt Kreditobergrenze

Ralf Daum und Jens Heiling: Potenziale des Electronic Government für Hochschulen und Studierende

Erik Peters: Auditing in Kanada

Roger W. Sonderegger: Steuerung von öffentlichen Unternehmen

Hildegard Pamme und Matthias Eßing:

Personalentwicklung in den Kommunen

Sven K. Mertens: IT-Sicherheitsinitiativen des

E-Government

Klaus Wirth: Innovationsmanagement in der öffentlichen Verwaltung

Axel Thomas: Medizinische Versorgungszentren

Andreas Gehlert und Karsten Halbauer: Temporale Aspekte in der Geschäftsprozessmodellierung in öffentlichen Verwaltungen 